\title{
Return to Paradise. Les dossiers oubliés : le fardeau de l'homme blanc de Jean GUIART
}

\section{Raymond MAYER}

\section{OpenEdition}

Journals

Édition électronique

URL : http://journals.openedition.org/jso/6452

DOI : $10.4000 /$ jso. 6452

ISSN : $1760-7256$

Éditeur

Société des océanistes

\section{Édition imprimée}

Date de publication : 15 décembre 2011

Pagination : 437-438

ISBN : 978-2-85430-031-4

ISSN : 0300-953x

\section{Référence électronique}

Raymond MAYER, "Return to Paradise. Les dossiers oubliés : le fardeau de l'homme blanc de Jean GUIART », Journal de la Société des Océanistes [En ligne], 133 | 2e semestre 2011, mis en ligne le 31 décembre 2011, consulté le 24 septembre 2020. URL : http://journals.openedition.org/jso/6452 ; DOI : https:// doi.org/10.4000/jso.6452

Ce document a été généré automatiquement le 24 septembre 2020.

(c) Tous droits réservés 


\title{
Return to Paradise. Les dossiers oubliés : le fardeau de l'homme blanc de Jean
}

\section{GUIART}

\author{
Raymond MAYER
}

\section{RÉFÉRENCE}

GUIART Jean, 2011. Return to Paradise. Les dossiers oubliés : le fardeau de l'homme blanc, Tahiti et Nouméa, Le Rocher-à-la-Voile, 4 photogr., bibliogr., addenda, 302 p.

1 L'auteur qui en est à publier deux ou trois livres par an dans la maison d'édition qu'il a fondée, pratique l'art du contrepoint dans une posture éthique que de nombreux lecteurs peuvent juger excessive et qui génère ainsi des avis plus ou moins bien partagés par d'autres. Cet ouvrage ne déroge pas à la règle. Empruntant son titre à un romancier américain de la fin de la guerre 1939-1945, celui-ci est un retour sur des thèmes déjà abordés à souhait dans ses livres antérieurs. Le style est rêche et les formules cinglantes à l'envi. L'allure est conversationnelle et ne craint pas de s'égarer dans d'infinies digressions qui ne sont sans doute pas inutiles pour ceux qui les découvrent pour la première fois, quitte à irriter ceux qui sont rompus aux classiques de l'Océanie.

2 Return to Paradiseest donc d'abord un retour sur le livre de l'écrivain américain James A. Michener qui portait ses réflexions sur l'après-guerre dans le Pacifique et le «retour » précisément de la colonisation. Jean Guiart charge la mule en faisant du paradis «le fardeau » de la colonisation et de tous les méfaits volontaires et involontaires qui grèvent les rapports non résolus entre acteurs endogènes « océaniens » et exogènes « blancs » du Pacifique. Sur la couverture du livre, une peinture caricaturale d'un portrait au bois de cerf semble davantage connoter la figure du cocu que celle de l'amateur de vahinés et de cocotiers. 
3 Le premier thème intitulé « Les données essentielles méconnues » (pp. 29-54) revient à tous les objets, outils et plantes qui fondent l'environnement tant social qu'écologique du Pacifique. Étoffes, bambou, fleur de tiaré, fruit à pain, patate douce, etc. jalonnent un parcours qui conduit le lecteur aux paysages construits, aux îles artificielles et aux marae. "L'Océanie est ainsi un laboratoire technique aussi bien que sociologique" conclut Jean Guiart en magnifiant l'esprit d'inventivité en toutes choses, là où elle lui semble manquer à nombre de chercheurs et d'analyses produites.

4 Il s'insurge au deuxième chapitre (pp.55-90) contre les pseudo-reconstructions du passé, qu'elles soient linguistiques, historiques ou anthropologiques, parce qu'elles continuent à légitimer une « pyramide raciale » où les Blancs occupent le sommet et les Mélanésiens la base. Ces reconstructions trahissent les fondamentaux des disciplines respectives, et simplifient outrancièrement la réalité dans le passé long autant que le passé court. Au fond les thèses et antithèses de Jean Guiart se réduisent à peu de choses : rééquilibrage mental de notre perception de la hiérarchie Mélanésie/Polynésie en faveur de la première ; prise en compte des systèmes de variantes dans l'examen des traditions orales; redéfinition d'un certain nombre de concepts appliqués sans discernement à la zone Pacifique.

5 Vient un troisième thème (pp. 91-132) qui lui tient tout autant à cœur: « De quoi la tradition orale parle-t-elle? » Le commentaire de corpus oraux est abondamment servi par le recours à des extraits significatifs en provenance de plusieurs cultures insulaires. Il prône l'ascension des érudits océaniens et donne des exemples de "poétique mélanésienne ", laquelle n'a "aucune raison de ne pas être tout aussi fertile qu'en Polynésie ». Dont acte.

6 Le quatrième thème, "Le miroir déformant» (pp.133-149), est réservé à la dénonciation de films coloniaux et au façonnage d'une opinion publique (voire scientifique) qui est engluée dans des clichés et des stéréotypes difficiles à éradiquer. La cinquième tête de chapitre, "Les tentatives d'organisation anti-coloniale spontanée " (pp. 151-181), est placée sous le signe de trois résistants du xxe siècle : Kabu et Yali pour la Papouasie Nouvelle-Guinée, ainsi que Paliau pour les îles de l'Amirauté. Ce chapitre est le seul illustré de trois photographies sur des figures de la résistance océanienne à la colonisation

7 La sixième partie des réflexions de Jean Guiart est dédiée aux mouvements «millénaristes?» (qualificatif significativement marqué d'un point d'interrogation) des Polynésiens et des Chinois à Tahiti. Le sujet chinois est traité sur le mode d'un thriller qui titille les aspirations mafieuses de certains réseaux de la diaspora chinoise et peut donner la chair de poule en évoquant la vision tentaculaire d'une extension sans limites de procédés réservés jusque-là à la Russie et à l'Italie! Le septième chapitre, "Guerres coloniales» (pp. 203-259), passe en revue, dans une quinzaine d'archipels, les conflits larvés, les insurrections historiques, ainsi que les émeutes et les coups d'État plus récents qui s'y déroulent.

8 Enfin «le soleil se lève à l'est " (pp. 261-269) est la nouvelle ombre portée par les puissances asiatiques sur leurs riverains du Pacifique, à la faveur du désengagement progressif des puissances occidentales dans la zone. Le pessimisme domine dans la vision d'avenir que tente Jean Guiart de la nouvelle donne.

9 Le meilleur du livre est probablement à la fin dans l'addenda (pp. 277-301) consacré au massacre de la grotte d'Ouvéa de 1988, par le compte rendu que dresse Jean Guiart du 
livre d'un colonel de gendarmerie (Alain Picard) en 2008, soit vingt ans après les faits. Jean Guiart tient un discours d'antithèse à rebours de toutes les déclarations officielles convenues: pour lui, la "poche protestante» de Gossanah a été victime des manipulations de la chefferie «catholique »; et ce sont ces manipulations qui ont abouti aux meurtres perpétrés sur les blessés par certains commandos dits d'élite de l'armée française sur ordre du président de la république française, François Mitterrand. Au moment où la même république vit toujours dans le négationnisme des massacres de « Français musulmans » du 17 octobre 1961 en plein cœur de Paris, il n'est pas interdit de penser que les thèses officiellement assénées ne sont pas forcément les plus respectables.

Par ailleurs, tout en reconnaissant la juste émotion suscitée par le film documentaire Tjibaou le pardon de Gilles Dagneau (2007), Jean Guiart conteste la version des interprétations délivrée par le film. Au terme d'une véritable investigation «archéologique » de la situation locale, Jean Guiart peut se permettre d'écrire cette phrase terrible :

«Tjibaou n'a pas été assassiné, il a été exécuté devant une foule passive et hostile, tout entière complice de cet acte de justice canaque remontant aux anciens âges, où le chef qui avait commis une faute grave était mis à mort. » (p. 297)

11 On reconnaît là la thèse déjà défendue dans son précédent livre de $2007 \mathrm{Du}$ sang sur le sable. Le vrai destin de Jean-Marie Tjibaou (compte rendu dans le Jso 129). Le débat reste à tout le moins entrouvert et, sans pour autant donner de blanc-seing à l'antithèse de Jean Guiart, on ne peut que renvoyer les historiens et les témoins muets de cette tragédie à leurs arguments scientifiques respectifs et à la dose d'opacité qui la recouvrira sans aucun doute à perpétuité. 